\title{
THE
}

UNIVERSITY

University of Rhode Island

OF RHODE ISLAND

DigitalCommons@URI

2-15-1979

\section{Can the Future Influence the Present?}

R. D. Driver

University of Rhode Island, rod_driver@uri.edu

Follow this and additional works at: https://digitalcommons.uri.edu/math_facpubs

Terms of Use

All rights reserved under copyright.

\section{Citation/Publisher Attribution}

Driver, R.D. (1978). Can the future influence the present? Physical Review D, 19(4), 1098.

Available at: http://dx.doi.org/10.1103/PhysRevD.19.1098

This Article is brought to you for free and open access by the Mathematics at DigitalCommons@URI. It has been accepted for inclusion in Mathematics Faculty Publications by an authorized administrator of DigitalCommons@URI.For more information, please contact digitalcommons-group@uri.edu. 


\title{
Can the future influence the present?
}

\author{
R. D. Driver \\ Department of Mathematics, University of Rhode Island, Kingston, Rhode Island 02881
}

(Received 10 April 1978)

\begin{abstract}
One widely accepted model of classical electrodynamics assumes that a moving charged particle produces both retarded and advanced fields. This formulation first appeared at least 75 years ago. It was popularized in the 1940's by work of Wheeler and Feynman. But the most fundamental question associated with the model has remained unanswered: When (if ever) does the two-body problem have a unique solution? The present paper gives an answer in one special case. Imagine two identical charged particles alone in the universe moving symmetrically along the $x$ axis. One is at $x(t)$ and the other is at $-x(t)$. Their motion is then governed by a system of functional differential equations involving both retarded and advanced arguments. This system together with the Newtonian "initial" data $x(0)=x_{0}>0$ and $x^{\prime}(0)=0$ has a unique solution for all time provided $x_{0}$ is sufficiently large. Perhaps the existence and uniqueness proof given for this special case will pave the way for more general results on this curious two-body problem.
\end{abstract}

\section{BACKGROUND}

If one assumes that the basic laws of physics must be symmetric with respect to time reversal, then the existence of retarded interactions between charged particles implies the existence also of advanced interactions.

This notion appeared as early as 1903 in a paper by Schwarzschild. In this model, charged particles influence each other via both retarded and advanced actions at a distance. The same model reappeared in the 1920's in the work of Tetrode, Page, Fokker, and others; and it finally became quite respectable after the famous papers of Wheeler and Feynman in 1945 and 1949 (Ref. 1) discussed the associated questions of causality.

But until the 1960's no progress was made on what may be considered the most fundamental question for this model-the two-body problem.

In 1963, Schild ${ }^{2}$ exhibited a special class of solutions of the two-body problem in which two particles of opposite sign move in concentric circular orbits in a plane. And, more recently, Andersen and von Baeyer ${ }^{3}$ have numerically computed solutions for the case of nearly circular plane orbits and for a case of one-dimensional motion.

The work of Schild showed that the two-body problem does have at least some solutions. But in the 15 years since Schild's work (and the 75 years since Schwarzschild's) the basic uniqueness question for this curious two-body problem has, to my knowledge, remained unanswered.

The question is: When does the two-body problem have a unique solution? In other words, what type of supplementary conditions, if any, could one impose in order that the two-body problem make sense?. Would a unique solution be ensured if one specified some type of boundary conditions, or appropriate trajectory segments (as suggested in Ref. 1), or initial values, or asymptotic conditions, or what?

Note that as soon as one imposes supplementary conditions in hopes of ensuring uniqueness, the question of existence becomes unknown again. So existence and uniqueness must be considered together.

As a prelude to studying this problem mathematically, a related but simpler problem was examined. In the simpler problem two charged particles are assumed to be influenced only by their mutual retarded interactions. This leads to a system of delay differential equations with unknown variable delays determined by the unknown trajectories. In general, for differential equations with time delays, one expects a unique solution to be determined for $t \geqslant 0$ if appropriate past histories (trajectories) are specified on some interval of the form $(\alpha, 0]$. Such results have been confirmed mathematically for the twobody problem in one space dimension, both without radiation reaction terms ${ }^{4}$ and with. ${ }^{5}$ Recently these results have been extended to three-dimensional motion without radiation reaction. ${ }^{6}$ It has also been found (to a mathematician's surprise) that, in one dimension, the trajectories are sometimes uniquely determined for $t \leqslant 0$ (and hence for $t \geqslant 0$ ) by their positions and velocities at $t=0$. See Driver, ${ }^{7}$ Zhdanov, ${ }^{8}$ and Hsing. ${ }^{9}$

Returning to the case of retarded and advanced interactions, it has been asserted ${ }^{10}$ that (even for the $n$-body problem) the trajectories are uniquely determined in case of distant collisions by the positions and velocities of all particles at $t=0$. Such a result would be surprising, for it is easily shown that a simple equation such as 


$$
x^{\prime}(t)=x(t-1)+x(t+1)
$$

has infinitely many solutions on $R$ satisfying infinitely many conditions at $t=0$, say,

$$
x(0)=0, \quad x^{\prime}(0)=0, \quad x^{\prime \prime}(0)=0, \ldots
$$

(see Ref. 7). And, more relevantly, Andersen and von Baeyer's calculations suggest that solutions of the electrodynamics two-body problem itself may not be uniquely determined by Newtonian data at $t=0$.

Nevertheless, this paper will show the following. Consider the special case of two identical (repelling) particles moving symmetrically in one space dimension. Then unique trajectories for all time are determined by the positions of the particles at the instant when both come to rest provided they are sufficiently far apart.

\section{THE PROBLEM}

For simplicity we consider only the special case of two identical charged particles moving symmetrically about the origin on the $x$ axis. At time $t$ one particle is at $x(t)>0$ and the other is at $-x(t)$.

The particle at $x(t)$ feels the retarded influence of the other particle, emitted at an instant $t-r(t)$, and the advanced influence (to be) emitted by the other particle at $t+q(t)$ (Fig. 1). The delay $r=r(t)$ and the advance $q=q(t)$ are the times required for a "light signal" to reach $x(t)$ from $-x(t-r(t))$ and from $-x(t+q(t))$, respectively. Thus they must satisfy the functional equations

$$
\operatorname{cr}(t)=x(t)+x(t-r(t))
$$

and

$$
c q(t)=x(t)+x(t+q(t))
$$

where $c$ is the speed of light.

The equations of motion [for the particle at $x(t)$ ] can now be expressed in terms of the interactions computed from the half-retarded plus half-advanced Lienard-Wiechert potentials together with the Lorentz force law. For economy of notation let us write $x, v, r$, and $q$ instead of $x(t), v(t)$, $r(t)$, and $q(t)$. Then if

$$
\begin{aligned}
& \left.x^{\prime}=c v \quad \text { (where }|v|<1\right), \\
& \frac{v^{\prime}}{\left(1-v^{2}\right)^{3 / 2}}=\frac{k}{r^{2}} \frac{1-v(t-r)}{1+v(t-r)}+\frac{k}{q^{2}} \frac{1+v(t+q)}{1-v(t+q)},
\end{aligned}
$$

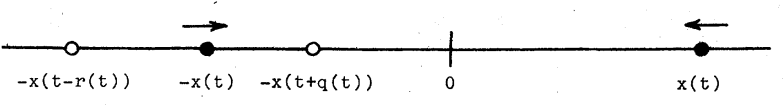

FIG. 1. Retarded, present, and advanced positions. where

$$
c r=x+x(t-r) \text { and } c q=x+x(t+q) .
$$

Here $k$ is a positive constant proportional to the product of the charges divided by the rest mass of one of them. (If the particles are electrons, $k c$ is half the classical electron radius.)

If the positions and velocities of the two particles are specified at $t=0$ a "point-data problem" can be described as follows.

Definition. Given $x_{0}>0$ and $v_{0} \in(-1,1)$, a solution on $R$ of Eqs. (1), (2), and (3) satisfying the "initial" conditions

$$
x(0)=x_{0} \text { and } v(0)=v_{0}
$$

is a function $\langle x, v\rangle: R \rightarrow(0, \infty) \times(-1,1)$ such that (1), (2), and (3) are satisfied on $R$ while $x(0)=x_{0}$ and $v(0)=v_{0}$.

This paper will treat only the special type of initial data

$$
x(0)=x_{0}>0, v(0)=0 .
$$

Note that since the two particles repel each other $(k>0)$, any solution must satisfy $v(t)=0$ for some instant $t$. We are simply calling that instant 0 and assuming that $x(0)$ is known.

Main theorem. If $x_{0}$ is sufficiently large, Eqs. (1), (2), and (3) have a unique solution on $R$ satisfying conditions (4).

The proof is given in Secs. IV and V below.

\section{A SIMPLE EXAMPLE}

Since Eqs. (1), (2), (3), and (4) represent a rather unprecedented mathematical problem, it may be worthwhile to begin by discussing a much simpler artificial illustrative example.

Consider the first-order differential equation with a constant delay $r>0$ and a constant advance $q>0$,

$$
x^{\prime}=f(t, x, x(t-r), x(t+q)),
$$

with initial condition

$$
x(0)=x_{0} .
$$

Let us assume that $f$ is continuous and satisfies

$$
\int_{-\infty}^{\infty}|f(t, 0,0,0)| d t<\infty
$$

plus the very restrictive Lipschitz-type condition

$$
\begin{aligned}
&|f(t, \xi, \eta, \zeta)-f(t, \tilde{\xi}, \tilde{\eta}, \tilde{\zeta})| \\
& \leqslant K(t) \max \{|\xi-\tilde{\xi}|,|\eta-\tilde{\eta}|,|\zeta-\tilde{\zeta}|\},
\end{aligned}
$$

where $K$ is continuous and

$$
\left|\int_{0}^{ \pm \infty} K(t) d t\right| \leqslant \alpha<1 .
$$


Then we can easily show that example $(\mathrm{E})$ has a unique solution on $R$ within the class of bounded functions:

Let

$$
S=\{g \in C(R, R): g(t) \text { is bounded on } R\} .
$$

Then $S$ is a complete metric space with the metric $d$ defined by

$$
d(g, \tilde{g})=\sup _{t \in R}|g(t)-\tilde{g}(t)|
$$

$$
\begin{aligned}
|(T g)(t)| & \leqslant\left|x_{0}\right|+\left|\int_{0}^{t} f(s, 0,0,0) d s\right|+\left|\int_{0}^{t}[f(s, g(s), g(s-r), g(s+q))-f(s, 0,0,0)] d s\right| \\
& \leqslant\left|x_{0}\right|+\int_{-\infty}^{\infty}|f(s, 0,0,0)| d s+\alpha B<\infty .
\end{aligned}
$$

So $T$ maps $S$ into $S$. Moreover, $T$ is a contraction mapping since, for any $g, \tilde{g} \in S$,

$$
\begin{aligned}
|(T g)(t)-(T \tilde{g})(t)| & \leqslant\left|\int_{0}^{t} K(s) d(g, \tilde{g}) d s\right| \\
& \leqslant \alpha d(g, \tilde{g}),
\end{aligned}
$$

which implies $d(T g, T \tilde{g}) \leqslant \alpha d(g, \tilde{g})$.

It follows that $T$ has a unique fixed point in $S$, and this is equivalent to showing that example (E) has a unique bounded solution.

(The above is a simple modification of results found by Polossuchin, Fite, ${ }^{11}$ and others. For further discussion and references see Ref. 7.)

But note that the assertion that example $(E)$ has a unique bounded solution does not mean that it has a unique solution. For instance, the equation

$$
x^{\prime}(t)=-b(-t) x(t-1)+b(t) x(t+1),
$$

where

$$
b(t)=\left\{\begin{array}{l}
0 \text { for } t \leqslant 0, \\
2 t e^{-2 t-1} \text { for } t \geqslant 0,
\end{array}\right.
$$

satisfies the hypotheses assumed for example (E). Thus it has a unique bounded solution satisfying $x(0)=x_{0}$. But, if $x_{0} \neq 0$, there is also an unbounded solution $x(t)=x_{0} e^{t 2}$. (This is a minor modification of an example of de Bruijn. ${ }^{12}$ )

Our task for the electrodynamics equations (1) -(4) is harder than the above because

(i) the electrodynamics equations are more complicated and they involve an unknown delay and an unknown advance,

(ii) the electrodynamics equations are autonomous, and so cannot satisfy such a convenient Lipschitz condition as we assumed for $f$ above, and for $g, \tilde{g} \in S$.

Now for $g \in S$ define $T g$ by

$$
(T g)(t)=x_{0}+\int_{0}^{t} f(s, g(s), g(s-r), g(s+q)) d s
$$

for $t \in R$.

Clearly $T g$ is a continuous function on $R$, and if $|g(t)| \leqslant B$, then (iii) we seek to prove a complete uniqueness theorem-not one which is restricted to a class of "bounded" solutions.

Despite these difficulties, the essence of the proof which follows will be the same as that used for example (E).

\section{PROPERTIES OF SOLUTIONS OF EQS. (1)-(4)}

If Eqs. (1)-(4) have a solution on $R$ it must satisfy certain conditions. We will obtain several of these conditions and then use some of them to describe a metric space $S$ in which solutions must lie. This space $S$ will be used in Sec. $V$ as the basis for a contraction mapping argument to complete the proof of the main theorem.

Lemma 1. Let $x$ be some differentiable function with $\left|x^{\prime}(t)\right|<c$ on $R$, and let Eqs. (3) have solutions $r(t)$ and $q(t)$ for all $t$. Then these solutions are unique and, letting $v=x^{\prime} / c$,

$$
r^{\prime}=\frac{v+v(t-r)}{1+v(t-r)} \text { and } q^{\prime}=\frac{v+v(t+q)}{1-v(t+q)} \text {. }
$$

[Note that in this lemma we do not assume that $x$ is in any way related to a solution of Eq. (2).]

Proof. If $r=r(t)$ and $\tilde{r}=\tilde{r}(t)$ satisfy the first of Eqs. (3), then if $r \neq \tilde{r}$ at some $t$

$$
\begin{aligned}
c|r-\tilde{r}| & =|x(t-r)-x(t-\tilde{r})| \\
& <c|r-\tilde{r}|,
\end{aligned}
$$

a contradiction. Thus $r=\tilde{r}$. (The uniqueness of $q$ is proved similarly.)

Equations (5) follow from the implicit function theorem. (See, for example, Landau, ${ }^{13}$ theorem 315.)

Lemma 2. If Eqs. (1)-(4) have a solution on $R$, 
then

$$
\begin{aligned}
& -1<v(t) \leqslant 0 \text { for } t \leqslant 0, \\
& 0 \leqslant v(t)<1 \text { for } t \geqslant 0,
\end{aligned}
$$

$$
\begin{aligned}
v v^{\prime} \leqslant & \frac{v v^{\prime}}{\left(1-v^{2}\right)^{1 / 2}} \\
\leqslant & \frac{k v}{r^{2}}[1-v(t-r)](1+v)\left(1-r^{\prime}\right) \\
& +\frac{k}{q^{2}} \frac{v+v(t+q)}{1-v(t+q)}\left(1-v^{2}\right) \\
\leqslant & \frac{4 k v}{r^{2}}\left(1-r^{\prime}\right)+\frac{k}{q^{2}} q^{\prime} \\
\leqslant & \frac{4 k c}{\left(x_{0}+x\right)^{2}} x^{\prime}-\frac{4 k v}{r^{2}} r^{\prime}+\frac{k}{q^{2}} q^{\prime} .
\end{aligned}
$$

and

$$
v(t-r) \leqslant v(t) \leqslant v(t+q) .
$$

Proof. These assertions all follow easily from the fact that $k>0$, and so $v^{\prime}(t)>0$ for all $t$.

The $a$ priori upper bound for $|v(t)|$ given in the next lemma is a key estimate enabling us to restrict the class of functions to be considered as possible solutions of Eqs. (1)-(4). Its proof depends on the observation that Eqs. (5) are equivalent to

$$
1-r^{\prime}=\frac{1-v}{1+v(t-r)}
$$

and

$$
1+q^{\prime}=\frac{1+v}{1-v(t+q)}
$$

the right-hand sides of which are very similar to some parts of Eq. (2).

Lemma 3. If Eqs. (1)-(4) have a solution on $R$, then

$$
|v(t)| \leqslant 3\left(k c / x_{0}\right)^{1 / 2} \text { for all } t .
$$

Proof. Consider the case $t \geqslant 0$. Equation (2) and the first of $\left(5^{\prime}\right)$ give

$$
\begin{aligned}
\frac{v v^{\prime}}{\left(1-v^{2}\right)^{3 / 2}}= & \frac{k v}{r^{2}} \frac{1-v(t-r)}{1-v}\left(1-r^{\prime}\right) \\
& +\frac{k}{q^{2}} \frac{v+v v(t+q)}{1-v(t+q)}
\end{aligned}
$$

Now invoke the inequalities

$$
1-r^{\prime}(t)>0, \quad|v(t-r)|<1,
$$

and

$$
0 \leqslant v(t) \leqslant v(t+q)<1
$$

plus the second equation of (5) to obtain

Integration from 0 to $t \geqslant 0$ gives

$$
\frac{1}{2} v^{2} \leqslant \frac{4 k c}{2 x_{0}}-4 k \int_{0}^{t} \frac{v(s) r^{\prime}(s)}{r^{2}(s)} d s+\frac{k}{q(0)} .
$$

Now, by the second mean value theorem for integrals (e.g., Landau, Ref. 13, theorem 405)

$$
\begin{aligned}
\int_{0}^{t} \frac{v(s) r^{\prime}(s)}{r^{2}(s)} d s & =v(t) \int_{\xi}^{t} \frac{r^{\prime}(s)}{r^{2}(s)} d s \\
& =v(t)\left[\frac{1}{r(\xi)}-\frac{1}{r(t)}\right]
\end{aligned}
$$

for some $\xi$ in $(0, t)$. Thus

$$
\frac{1}{2} v^{2} \leqslant \frac{2 k c}{x_{0}}+\frac{4 k}{r(t)}+\frac{k}{q(0)} \leqslant \frac{9 k c}{2 x_{0}} .
$$

This together with the similar inequality for $t \leqslant 0$ completes the proof.

In the "nonrelativistic case" the exact bound for $|v(t)|$ is

$$
u_{0} \equiv\left(k c / x_{0}\right)^{1 / 2} \text {. }
$$

The next result sharpens the estimate of lemma 3 to approximately that bound if $x_{0} \gg k c$. This refinement would not be necessary in order to prove the main theorem; but the improved estimate is useful when one tries to determine what the condition " $x_{0}$ is sufficiently large" really means.

Lemma 4. Assume $x_{0}>36 k c$ and that Eqs. (1)(4) have a solution on $R$. Then $|v(t)| \leqslant u$ for all $t$, where $u$ is any solution in $\left(0, \frac{1}{2}\right]$ of the inequality

$$
u^{2} \geq \frac{1+u}{1-u}\left(1+u+\frac{1}{2} u^{2}\right) u_{0}^{2} .
$$

Remark. If $x_{0} \gg k c$, one can take $u$ just slightly larger than $u_{0}$. For example, if $x_{0}=100 k c$ so that $u_{0}=0.1$, Lemma 4 gives $|v(t)| \leqslant u=0.12-\mathrm{a}$ considerable improvement over the estimate $|v(t)| \leqslant 0.3$ given by Lemma 3 .

Proof of Lemma 4. Let $u_{1}=\sup _{t \in R}|v(t)|$. Thus $u_{1} \leqslant 3 u_{0}<\frac{1}{2}$. Then from (3), $c r \geqslant 2 x-c u_{1} r$ so that

$$
r \geqslant \frac{2 x}{c\left(1+u_{1}\right)}
$$


Now for $t \geqslant 0$, (3) yields

$$
q=\frac{x+x(t+q)}{c} \geqslant \frac{2 x}{c},
$$

so from Eq. (2) one finds

$$
\begin{aligned}
v v^{\prime} & \leqslant \frac{v v^{\prime}}{\left(1-v^{2}\right)^{3 / 2}} \\
& \leqslant \frac{k c}{4 x^{2}} x^{\prime}\left[\frac{\left(1+u_{1}\right)^{3}}{1-u_{1}}+\frac{1+u_{1}}{1-u_{1}}\right] \text { for } t \geqslant 0 .
\end{aligned}
$$

Thus, by integration from 0 to $t$,

$$
\frac{t}{2} v^{2} \leqslant \frac{\left(1+u_{1}\right)\left(2+2 u_{1}+u_{1}^{2}\right)}{1-u_{1}} \frac{k c}{4 x_{0}} \text { for } t \geqslant 0 .
$$

This together with an analogous calculation for $t \leqslant 0$ shows that

$$
u_{1}^{2} \leqslant \frac{1+u_{1}}{1-u_{1}}\left(1+u_{1}+\frac{1}{2} u_{1}^{2}\right) \frac{k c}{x_{0}} \equiv f\left(u_{1}\right) \frac{k c}{x_{0}},
$$

or $f\left(u_{1}\right) / u_{1}^{2} \geqslant x_{0} / k c$. Now

$$
\begin{aligned}
\frac{d}{d u}\left[\frac{f(u)}{u^{2}}\right] & =\frac{-2+u+4 u^{2}+2 u^{3}}{u^{3}(1-u)^{2}} \\
& <0 \text { for } 0<u \leqslant \frac{1}{2}
\end{aligned}
$$

So if $0<u \leqslant \frac{1}{2}$ with $f(u) / u^{2} \leqslant x_{0} / k c$ it follows that $u \geqslant u_{1}$.

In addition to the upper bounds for $|v(t)|$, we will also need an upper bound for $\left|v^{\prime}(t)\right|$.

Lemma 5. Let Eqs. (1)-(4) have a solution on
$R$ with $|v(t)| \leqslant u<1$. Then for all $t$

$$
x(t) \geqslant c p(t) \equiv c \max \left\{\frac{x_{0}}{c}, a u_{0}|t|\right\}
$$

and

$$
\left|v^{\prime}(t)\right| \leqslant \frac{b k}{4 p^{2}(t)},
$$

where

$$
\begin{aligned}
& u_{0}=\left(\frac{k c}{x_{0}}\right)^{1 / 2}, \\
& a=\frac{1-u^{2}}{1.2}, b=\frac{2(1+u)^{3}}{1-u} .
\end{aligned}
$$

Proof. To obtain a decreasing upper bound for $\left|v^{\prime}(t)\right|$ from Eq. (2), one must first find an increasing lower bound for $x(t)$, and hence for $r(t)$ and $q(t)$. The argument will be similar to that used by Zhdanov ${ }^{8}$ for a problem involving only retarded interactions.

From Eqs. (3), $c q=2 x+c v(\xi) q$ for some $\xi \in(t, t+q)$. So

$$
q=\frac{2 x}{c-c v(\xi)} \leqslant \frac{2 x}{c[1-v(t+q)]} .
$$

Similarly

$$
r \leqslant \frac{2 x}{c[1+v(t-r)]} .
$$

Substitute these estimates into Eq. (2) to find

$$
\frac{v^{\prime}}{\left(1-v^{2}\right)^{3 / 2}} \geqslant \frac{k c^{2}}{4 x^{2}}\left[1-v^{2}(t-r)\right]+\frac{k c^{2}}{4 x^{2}}\left[1-v^{2}(t+q)\right] \geqslant \frac{k c^{2}\left(1-u^{2}\right)}{2 x^{2}} .
$$

Thus for $t \geqslant 0$

$$
\int_{0}^{t} \frac{k c\left(1-u^{2}\right)}{2 x^{2}(s)} x^{\prime}(s) d s \leqslant \int_{0}^{t} \frac{v(s) v^{\prime}(s)}{\left[1-v^{2}(s)\right]^{3 / 2}} d s=\frac{1-\left(1-v^{2}\right)^{1 / 2}}{\left(1-v^{2}\right)^{1 / 2}} .
$$

So

$$
\frac{k c\left(1-u^{2}\right)}{2}\left(\frac{1}{x_{0}}-\frac{1}{x}\right) \leqslant \frac{v^{2}}{\left(1-v^{2}\right)^{1 / 2}+1-v^{2}} \leqslant \frac{v^{2}}{2\left(1-u^{2}\right)} .
$$

This becomes

$$
v^{2}+\frac{k c\left(1-u^{2}\right)^{2}}{x} \geq \frac{k c\left(1-u^{2}\right)^{2}}{x_{0}} \equiv E,
$$

or

$$
v^{2}+E \frac{x_{0}}{x} \geqslant E
$$

So (for $t \geqslant 0) x^{\prime}=c v \geqslant c\left[E\left(1-x_{0} / x\right)\right]^{1 / 2}$, which yields

$$
\sqrt{E} c t \leqslant \int_{x_{0}}^{x} \frac{1}{\left(1-x_{0} / z\right)^{1 / 2}} d z=x f\left(x_{0} / x\right),
$$

where

$$
f(y) \equiv(1-y)^{1 / 2}+y \ln \frac{(1-y)^{1 / 2}+1}{y^{1 / 2}}
$$


for $0<y \leqslant 1$. We want to replace $f\left(x_{0} / x\right)$ by its maximum. Computing $f^{\prime}(y)$ and $f^{\prime \prime}(y)$ one finds that $f^{\prime \prime}(y)$ $<0$ and that $f^{\prime}(0.305)>0$ while $f^{\prime}(0.306)<0$. So the maximum of $f(y)$ must occur between 0.305 and 0.306 , and we find $f(y) \leqslant 1.2$. Thus

$\sqrt{E} c t \leqslant 1.2 x$ for $t \geqslant 0$.

For $t \leqslant 0$ similar calculations give $-\sqrt{E} c t \leqslant 1.2 x$, and these results together yield $x(t) \geqslant(1 / 1.2) \sqrt{E} c|t|$ $=a u_{0} c|t|$. So $x(t) \geqslant c p(t)$.

To prove the remaining inequality in (7), note from Eqs. (3) that $c r \geqslant 2 x-c u r$ and $c q \geqslant 2 x-c u q$, or

$$
r \geqslant \frac{2 x}{c(1+u)} \text { and } q \geqslant \frac{2 x}{c(1+u)} \text { for all } t \text {. }
$$

Apply these in Eq. (2) to find

$$
\left|v^{\prime}(t)\right| \leqslant \frac{2 k c^{2}}{4 x^{2}} \frac{(1+u)^{3}}{1-u} \leqslant \frac{b k}{4 p^{2}(t)} \text { for all } t \text {. }
$$

It now follows that if $\langle x, v\rangle$ is a solution of Eqs. (1)-(4) on $R$ with $|v(t)| \leqslant u<1$, then $v$ belongs to the space

$$
S=\left\{g \in C(R, R): g(0)=0,|g(t)| \leqslant u, \text { and whenever } t_{2} \leqslant t_{1} \quad 0 \leqslant g\left(t_{1}\right)-g\left(t_{2}\right) \leqslant \frac{b k\left(t_{1}-t_{2}\right)}{4 \min \left\{p^{2}\left(t_{1}\right), p^{2}\left(t_{2}\right)\right\}}\right\} \text {, }
$$

where $p$ and $b$ are defined in (7) and (8). Note that $S$ is a complete metric space with the metric $d$ defined by

$$
d(g, \tilde{g})=\sup _{t \in R}|g(t)-\tilde{g}(t)| \text { for } g, \tilde{g} \in S .
$$

\section{PROOF OF THE MAIN THEOREM}

To prove the main theorem (stated in Sec. II) we shall define a mapping $T$ of $S$ into $S$ such that $T v=v$ for $v \in S$ if and only if $\langle x, v\rangle$ is a solution of Eqs. (1)-(4) when

$$
x(t) \equiv x_{0}+\int_{0}^{t} c v(s) d s .
$$

Then we shall show that $T$ is a contraction mapping provided $x_{0}$ is sufficiently large.

There are several ways in which one might attempt to define a mapping $T$ on $S$ such that $T v=v$ when $\langle x, v\rangle$ is a solution of Eqs. (1)-(4). But the most obvious choices for $T$ do not seem to work, that is, they fail to map all of $S$ into $S$. The construction used below was essentially invented by $\mathrm{Hsing}^{9}$ for the case when only retarded interactions are considered.

Definition of a Mapping $T$. For $g \in S$ define $T g=v$ where $\langle x, v, r, q\rangle$ is the unique solution of the system of ordinary differential equations

$$
\begin{aligned}
& x^{\prime}=c v, \\
& \frac{v^{\prime}}{\left(1-v^{2}\right)^{3 / 2}}=\frac{k}{r^{2}} \frac{1-g(t-r)}{1+g(t-r)}+\frac{k}{q^{2}} \frac{1+g(t+q)}{1-g(t+q)},
\end{aligned}
$$

$$
r^{\prime}=\frac{2 v-g+g(t-r)}{1+g(t-r)},
$$

and

$$
q^{\prime}=\frac{2 v-g+g(t+q)}{1-g(t+q)},
$$

with initial conditions

$x(0)=x_{0}, \quad v(0)=0, \quad r(0)=r_{0}$, and $q(0)=q_{0}$,

where

$c r_{0}=2 x_{0}+\int_{0}^{-r} c g(s) d s, \quad c q_{0}=2 x_{0}+\int_{0}^{q} c g(s) d s$.

Note that the functional equation for $r_{0}$ in (4a) does have a unique solution since $c \rho-\int_{0}^{-\rho} c g(s) d s$ is a differentiable function of $\rho$ with derivative $c+c g(-\rho) \geqslant c(1-u)>0$. Similarly one shows that the functional equation for $q_{0}$ has a unique solution. It also follows easily that $r_{0}$ and $q_{0}$ are positive.

The motivation for Eqs. (5a) is clarified by the following lemma showing that Eqs. (5a) together with their initial conditions in (4a) are equivalent to a pair of equations similar to (3).

Lemma 6. Let $g$ be a continuous function with $|g(t)| \leqslant u<1$ on $R$ and let $x$ be positive and continuously differentiable with $x^{\prime}(t)=c v(t)$ on $(\alpha, \beta)$, where $\alpha<0<\beta$. Then $r$ and $q$ satisfy Eqs. (5a) on $(\alpha, \beta)$ with $r(0)=r_{0}$ and $q(0)=q_{0}$ as in (4a) if and only if

$$
c r=2 x+\int_{t}^{t-r} \operatorname{cg}(s) d s
$$

and

$$
c q=2 x+\int_{t}^{t+q} \operatorname{cg}(s) d s
$$


on $(\alpha, \beta)$. Moreover, each of the equations in (3a) has a unique solution ( $r$ or $q$ ) and these solutions are positive.

Proof. Assume that $r$ and $q$ satisfy Eqs. (5a) on $(\alpha, \beta)$ together with the initial conditions of (4a). Then, from (5a),

$$
c r^{\prime}=2 c v-c g+c g(t-r)\left(1-r^{\prime}\right)^{\circ}
$$

and

$$
c q^{\prime}=2 c v-c g+c g(t+q)\left(1+q^{\prime}\right) .
$$

Now integrate these from 0 to $t \in(\alpha, \beta)$ and invoke the appropriate equations from (4a) to obtain (3a). Conversely, if Eqs. (3a) hold on $(\alpha, \beta)$, then Eqs. (5a) follow from the implicit function theorem.

The existence, uniqueness, and positivity of the solution of the first of Eqs. (3a) follow from the fact that $c \rho-\int_{t}^{t-\rho} \operatorname{cg}(s) d s$ is a differentiable function of $\rho$ with derivative

$$
c+c g(t-\rho) \geqslant c-c u>0 .
$$

The next lemma asserts that "fixed points" of the mapping $T$ yield solutions of Eqs. (1)-(4) and vice versa.

Lemma 7. Let $g \in S$ (with some $u<1$ ). Then $T g=g$ on $R$ if and only if $\langle x, g\rangle$ with

$$
x(t) \equiv x_{0}+\int_{0}^{t} \operatorname{cg}(s) d s
$$

is a solution of Eqs. (1)-(4) on $R$.

Proof. If $g=T g(=v)$ on $R$, then (1a), (2a), (3a), and (4a) yield Eqs. (1)-(4) on $R$. Conversely, if $\langle x, g\rangle$ is a solution of Eqs. (1)-(4) on $R$ with $x$ defined by Eq. (10), then $x^{\prime}=c g$, so that Eqs. (3) give (3a). Since $r$ and $q$ are uniquely determined by (3a), Eqs. (2) and (2a) show that $\left(1-v^{2}\right)^{-3 / 2} v^{\prime}$ $=\left(1-g^{2}\right)^{-3 / 2} g^{\prime}$ which, together with $v(0)=0=g(0)$, implies $v(t)=g(t)$, that is, $T g=g$.

Lemma 8. Let $x_{0} \geqslant 36 k c$, let $u$ be a solution of inequality (6) with $0<u<\frac{1}{2}$, and let $S$ be defined by (9). Then $T$ maps $S$ into $S$.

Proof. Let $g \in S$. Then Eqs. (1a), (2a), (5a), and (4a) will have a unique noncontinuable solution $\langle x, v, r, q\rangle$ on some interval $(\alpha, \beta)$ where $\alpha<0<\beta$. If we can show that $r$ and $q$ are bounded away from 0 and $|v| \leqslant u$ (or merely $|v| \leqslant 1$ ) on $(\alpha, \beta)$, then it will follow that $(\alpha, \beta)=R$.

Since $v(t) \geqslant 0$ for $0 \leqslant t<\beta$ and $v(t) \leqslant 0$ for $\alpha<t \leqslant 0$, $x(t) \geqslant x_{0}>0$ for $\alpha<t<\beta$. From (3a), $c r \geqslant 2 x-c u r$ and $c q \geqslant 2 x-c u q$ so that

$$
r \geqslant \frac{2 x}{c(1+u)} \geqslant \frac{2 x_{0}}{c(1+u)}>0
$$

and

$$
q \geqslant \frac{2 x}{c(1+u)} \geqslant \frac{2 x_{0}}{c(1+u)}>0
$$

for all $t$ in $(\alpha, \beta)$. More specifically, for $0 \leqslant t<\beta$ (3a) gives $c q \geqslant 2 x$, and so

$$
\begin{aligned}
v v^{\prime} & \leqslant \frac{v v^{\prime}}{\left(1-v^{2}\right)^{3 / 2}} \\
& \leqslant \frac{k c}{4 x^{2}}\left[\frac{(1+u)^{3}}{1-u}+\frac{1+u}{1-u}\right] x^{\prime}
\end{aligned}
$$

for $0 \leqslant t<\beta$. Integration from 0 to $t$ yields

$$
\begin{aligned}
v^{2} & \leqslant \frac{k c}{x_{0}} \frac{1+u}{1-u}\left(1+u+\frac{1}{2} u^{2}\right) \\
& \leqslant u^{2} \text { for } 0 \leqslant t<\beta .
\end{aligned}
$$

The same result is found for $\alpha<t \leqslant 0$. Thus $(\alpha, \beta)$ $=R$ and $|v(t)| \leqslant u$ for all $t$.

If we can now show that $\left|v^{\prime}(t)\right| \leqslant b k / 4 p^{2}(t)$ as in (7), it will follow that $v \in S$ and, since $v=T g, T$ maps $S$ into $S$. The following argument is analogous to the proof of Lemma 5 .

From Eqs. (3a), $c q=2 x+c g(\xi) q$ for some $\xi \in(t, t+q)$. So

$$
q=\frac{2 x}{c-c g(\xi)} \leqslant \frac{2 x}{c[1-g(t+q)]},
$$

and similarly

$$
r \leqslant \frac{2 x}{c[1+g(t-r)]} \text {. }
$$

Substitute these estimates into Eq. (2a) to find

$$
\frac{v^{\prime}}{\left(1-v^{2}\right)^{3 / 2}} \geqslant \frac{k c^{2}\left(1-u^{2}\right)}{2 x^{2}} \text { on } R \text {. }
$$

Now the proof proceeds word for word as in Lemma 5 to the estimate

$$
x(t) \geqslant c p(t) \text { for all } t .
$$

Using (11) and (13) in Eq. (2a), one then finds $\left|v^{\prime}(t)\right| \leqslant b k / 4 p^{2}(t)$.

The following lemma is the final step in the proof of the main theorem (stated in Sec. II).

Lemma 9. If $x_{0}$ is sufficiently large, there is a solution $u>0$ of inequality (6) such that $T$ is a contraction mapping of $S$ into $S$ [where $S$ is defined by (9)].

Proof. As a preliminary restriction let $x_{0} \geqslant 4500 k c$. Then $u_{0} \leqslant 0.01491$, and one can choose a solution $u$ of inequality (6) such that

$0<u \leqslant 1.0232 u_{0} \leqslant 0.01526$,

and hence

$$
0.8331 \leqslant a \leqslant \frac{1}{1.2} \text { and } b \leqslant 2.126 \text {. }
$$

Assume this has been done, and let $S$ be defined by (9).

Let $g, \tilde{g} \in S$ and let $\langle v, x, r, q\rangle$ and $\langle\tilde{v}, \tilde{x}, \tilde{r}, \tilde{q}\rangle$ be the solutions of Eqs. (1a), (2a), (5a), and (4a) using $g$ and $\tilde{g}$, respectively.

We would like to show that $d(T g, T \tilde{g}) \leqslant \alpha d(g, \tilde{g})$ for some $\alpha<1$. Let us estimate [writing $r$ and $q$ in. place of $r(s)$ and $q(s)]$ 


$$
\begin{aligned}
|v-\tilde{v}| & \leqslant\left|\frac{v}{\left(1-v^{2}\right)^{1 / 2}}-\frac{\tilde{v}}{\left(1-\tilde{v}^{2}\right)^{1 / 2}}\right| \\
& =\left|\int_{0}^{t}\left[\frac{v^{\prime}(s)}{\left[1-v^{2}(s)\right]^{3 / 2}}-\frac{\tilde{v}^{\prime}(s)}{\left[1-\tilde{v}^{2}(s)\right]^{3 / 2}}\right] d s\right| \\
& \leqslant\left|\int_{0}^{t} k\right| \frac{1}{r^{2}} \frac{1-g(s-r)}{1+g(s-r)}-\frac{1}{\tilde{r}^{2}} \frac{1-\tilde{g}(s-\tilde{r})}{1+\tilde{g}(s-\tilde{r})}+\frac{1}{q^{2}} \frac{1+g(s+q)}{1-g(s+q)}-\frac{1}{\tilde{q}^{2}} \frac{1+\tilde{g}(s+\tilde{q})}{1-\tilde{g}(s+\tilde{q})}|d s| \\
& \leqslant \mid \int_{0}^{t} k\left[\frac{1+u}{1-u}\left|\frac{1}{r^{2}}-\frac{1}{\tilde{r}^{2}}\right|+\frac{1}{r^{2}} \frac{2}{(1-u)^{2}}|g(s-r)-\tilde{g}(s-\tilde{r})|\right. \\
& \left.\quad+\frac{1+u}{1-u}\left|\frac{1}{q^{2}}-\frac{1}{\tilde{q}^{2}}\right|+\frac{1}{q^{2}} \frac{2}{(1-u)^{2}}|g(s+q)-\tilde{g}(s+\tilde{q})|\right] d s \mid .
\end{aligned}
$$

To simplify this further, we will need suitable upper estimates for $|r(s)-\tilde{r}(s)|$ and $|q(s)-\tilde{q}(s)|$ plus lower estimates for $p(s-r(s))$ and similar terms.

From the first of Eqs. (3a),

$$
\begin{aligned}
|r(s)-\tilde{r}(s)| & =\left|2 \int_{0}^{s}[v(\tau)-\tilde{v}(\tau)] d \tau+\int_{s}^{s-r} g(\tau) d \tau-\int_{s}^{s-\tilde{r}} \tilde{g}(\tau) d \tau\right| \\
& \leqslant 2\left|\int_{0}^{s}[v(\tau)-\tilde{v}(\tau)] d \tau\right|+r d(g, \tilde{g})+u|r(s)-\tilde{r}(s)| .
\end{aligned}
$$

For definiteness, let $s \geqslant 0$ and introduce

$$
w(s) \equiv \max _{0 \leqslant \tau \leqslant s}|v(\tau)-\tilde{v}(\tau)|
$$

Then

$$
|r(s)-\tilde{r}(s)| \leqslant \frac{1}{1-u}\left[r(s) d(g, \tilde{g})+\frac{2 s}{1-u} w(s)\right] .
$$

[The inequality also holds if one replaces $r(s)$ by $\tilde{r}(s)$ on the right-hand side.] Since by (12) $r(s)$ $\leqslant 2\left(x_{0}+c u s\right) / c(1-u)$, inequality $(16)$ also yields

$$
|r(s)-\tilde{r}(s)| \leqslant \frac{2}{(1-u)^{2}}\left[\left(\frac{x_{0}}{c}+u s\right) d(g, \tilde{g})+s w(s)\right]
$$

for $s \geqslant 0$. Analogous estimates hold for $|q(s)-\tilde{q}(s)|$.

To estimate $s-r(s)$, note from (5a) that

$$
1-r^{\prime}=\frac{1-2 v+g}{1+g(t-r)} \geqslant \frac{1-2 u}{1+u}>0 \text {. }
$$

So if $s \geqslant 0$

$$
|s-r(s)| \geqslant s-r(s) \geqslant-r_{0}+\frac{1-2 u}{1+u} s \geqslant-\frac{2 x_{0}}{c(1-u)}+\frac{1-2 u}{1+u}|s| .
$$

[When $s \leqslant 0$ we get the stronger estimate $|s-r(s)| \geqslant|s|$.] So, from (7),

$$
\begin{aligned}
2 p(s-r(s)) & \geqslant \frac{x_{0}}{c}+a u_{0}|s-r(s)| \\
& \geqslant \frac{x_{0}}{c}\left(1-\frac{2 a u_{0}}{1-u}\right)+\frac{1-2 u}{1+u} a u_{0}|s| \text { (for all } s \text { ). }
\end{aligned}
$$

The same inequality holds when $s-r(s)$ is replaced by $s-\tilde{r}(s), s+q(s)$, or $s+\tilde{q}(s)$. 
As inequalities (16), (17), and (18) are applied to (15), the statements will be simplified further by use of the numerical values in (14). Thus (18) yields

$$
2 p(s-r(s)) \geqslant \frac{1}{c}\left(0.97 x_{0}+0.79 c u_{0}|s|\right) \text { for all } s .
$$

Typical terms in the integrand on the right-hand side of (15) now reduce as follows. Since $r(s) \geqslant 2 p(s) /$ $(1+u) \geqslant 2 a u_{0}|s| /(1+u)$, (16) gives

$$
\begin{aligned}
\frac{1+u}{1-u}\left|\frac{1}{r^{2}}-\frac{1}{\tilde{r}^{2}}\right| & =\frac{1+u}{1-u}\left(\frac{1}{\tilde{r}^{2}} \frac{|r-\tilde{r}|}{r}+\frac{1}{r^{2}} \frac{|r-\tilde{r}|}{\tilde{r}}\right) \\
& \leqslant\left[\frac{1+u}{(1-u)^{2}} d(g, \tilde{g})+\frac{(1+u)^{2}}{(1-u)^{3}} \frac{w(s)}{a u_{0}}\right]\left(\frac{1}{r^{2}}+\frac{1}{\tilde{r}^{2}}\right) \\
& \leqslant\left[1.047 d(g, \tilde{g})+1.296 \frac{1}{u_{0}} w(s)\right]\left(\frac{1}{r^{2}}+\frac{1}{\tilde{r}^{2}}\right),
\end{aligned}
$$

while inequalities $(17),\left(18^{\prime}\right)$, and $p(s-r) \geqslant x_{0} / c$ yield

$$
\begin{aligned}
\frac{2}{r^{2}(1-u)^{2}}|g(s-r)-\tilde{g}(s-\tilde{r})| & \leqslant \frac{2}{r^{2}(1-u)^{2}}\left(d(g, \tilde{g})+\frac{b k|r-\tilde{r}|}{4 \min \left\{p^{2}(s-r), p^{2}(s-\tilde{r})\right\}}\right) \\
& \leqslant\left[2.0625 d(g, \tilde{g})+\frac{2 b k c}{(1-u)^{4} x_{0}}\left(\frac{x_{0}+1.03 c u_{0} s}{0.97 x_{0}+0.79 c u_{0} s} d(g, \tilde{g})+\frac{w(s)}{0.79 u_{0}}\right)\right] \frac{1}{r^{2}} \\
& \leqslant[2.064 d(g, \tilde{g})+0.086 w(s)] \frac{1}{r^{2}}
\end{aligned}
$$

Using these (and analogous inequalities), (15) yields for $t \geqslant 0$

$$
\begin{aligned}
w(t) \leqslant & d(g, \bar{g}) \int_{0}^{t}\left[3.111\left(\frac{k}{r^{2}}+\frac{k}{q^{2}}\right)+1.047\left(\frac{k}{\tilde{r}^{2}}+\frac{k}{\tilde{q}^{2}}\right)\right] d s \\
& +\int_{0}^{t}\left[\left(\frac{1.296}{u_{0}}+0.086\right)\left(\frac{k}{r^{2}}+\frac{k}{q^{2}}\right)+\frac{1.296}{u_{0}}\left(\frac{k}{\tilde{r}^{2}}+\frac{k}{\tilde{q}^{2}}\right)\right] w(s) d s .
\end{aligned}
$$

This inequality can be solved by use of the Gronwall-Reid lemma together with the observation from Eq. (2a) that for all $t \geqslant 0$,

$$
\begin{aligned}
\int_{0}^{t}\left(\frac{k}{r^{2}}+\frac{k}{q^{2}}\right) d s & \leqslant \int_{0}^{t} \frac{1+u}{1-u} \frac{v^{\prime}(s)}{\left[1-v^{2}(s)\right]^{3 / 2}} d s \\
& \leqslant \frac{1+u}{1-u} \frac{u}{\left(1-u^{2}\right)^{1 / 2}} \leqslant 1.0551 u_{0} .
\end{aligned}
$$

Thus,

$$
\begin{aligned}
w(\infty) & \leqslant d(g, \tilde{g}) 4.39 u_{0} \exp \left(2.735+0.091 u_{0}\right) \\
& \leqslant 67.74 u_{0} d(g, \tilde{g}) .
\end{aligned}
$$

This together with a completely analogous calculation for $t \leqslant 0$ shows that

$$
d(T g, T \tilde{g}) \leqslant 67.74 u_{0} d(g, \tilde{g}) .
$$

So $T$ is a contraction mapping if

$$
x_{0} \geqslant(67.74)^{2} k c=4589 k c \text {. }
$$

Remark. The proof of Lemma 9 provides a stronger result than was stated: Let the two particles be electrons, so that $k c$ is half the classical electron radius. Then the above proof shows that a unique solution exists for the two- body problem represented by Eqs. (1), (2), (3), and (4) provided the separation of the particles at $t=0$-namely $2 x_{0}$-is at least 4600 electron radii, or $1.3 \times 10^{-9} \mathrm{~cm}$.

This does not mean that the problem is ill posed for smaller separations. In fact one could easily extract somewhat sharper estimates from the calculations given here. However, anything like an order-of-magnitude improvement, if such is possible, would probably require more basic changes in the proof.

The sufficient separation of 4600 electron radii established here is a big improvement over the values which sometimes come out of such proofs-namely large multiples of the radius of the universe.

\section{DISCUSSION}

The question posed in the title of this paper may be impossible to answer since it may eventually reduce to a matter of interpretation. If the day ever comes when we are confident that we know a law of electrodynamics which exactly predicts the true behavior of interacting particles, per- 
haps that law will have several mathematically equivalent forms-e.g., action at a distance, interaction via fields, and a minimum principle. Then the choice will be a matter of convenience or taste, and perhaps only one of the equivalent forms will explicitly involve an "advanced interaction."

However, one can (and should) always ask whether a mathematical model makes sense.

The various mathematical models in use today for electrodynamics (or other physical processes) are presumably approximations at best to the "true" laws. So knowledge or intuition about the answers to certain questions in the real world does not predict the answers to the corresponding questions for the models. A model must be tested independently to see that the questions even have answers and that the answers are acceptable.

The present paper has begun those tests for

1J. A. Wheeler and R. P. Feynman, Rev. Mod. Phys. 17, 157 (1945); 21, 425 (1949).

${ }^{2}$ A. Schild, Phys. Rev. 131, 2762 (1963).

${ }^{3}$ C. M. Andersen and H. C. von Baeyer, Phys. Rev. D $\underline{5}$, 802 (1972); 5 , 2470 (1972).

${ }^{4}$ R. D. Driver, Ann. Phys. (N.Y.) 21, 122 (1963); R. D. Driver and M. J. Norris, ibid. $\overline{42}, 347$ (1967); S. P. Travis, SIAM J. Appl. Math. 28, 611 (1975).

${ }^{5}$ D. K. Hsing and R. D. Driver, Technical Report No. 61 , Dept. of Math., Univ. of Rhode Island, 1975 (unpublished); also (a special case), in Dynamical Systems, Proceedings of a University of Florida International Symposium (Academic, New York, 1977), pp. 427430.

${ }^{6}$ R. D. Driver, Technical Report No. 60, Dept. of Math., Univ. of Rhode Island, 1975; published (in Russian), in one model. A more precise title for this paper would have been: "An existence and uniqueness theorem for the classical relativistic model of two electrons in one-dimensional motion with half-retarded-half-advanced interactions." Clearly much more remains to be done. I hope that it will be possible to extend (or contradict) the existence and uniqueness assertions of this paper for the case of unsymmetric motion in one dimension, and then for motion in two or three dimensions.

\section{ACKNOWLEDGMENTS}

I am grateful to H. C. von Baeyer, R. N. Buchal, J. L. Synge, and J. A. Yorke for valuable suggestions and discussions regarding this problem. This research was supported by AFOSR Grant No. 77-3397.

Differencial'nye Uravnenija s Otklonjajuscimsja Argumentom (Naukova Dumka, Kiev, 1977), pp. 113-127.

${ }^{7}$ R. D. Driver, Phys. Rev. 178, 2051 (1969); and in Dynamical Systems, An International Symposium (Academic, New York, 1976), Vol. 2, pp. 115-121.

${ }^{8}$ V. I. Z hdanov, Int. J. Theor. Phys. 15, 157 (1976).

${ }^{9}$ D. K. Hsing, Phys. Rev. D 16, 974 (1977).

${ }^{10} \mathrm{H}$. Van Dam and E. P. Wigner, Phys. Rev. 142,838 (1966).

${ }^{11} \mathrm{O}$. Polossuchin, inaugural dissertation, Universität Zürich, 1910 (unpublished); W. B. Fite, Trans. Am. Math. Soc. 22, 311 (1921).

${ }^{12}$ N. G. de Bruijn, Am. J. Math. 71, 313 (1949).

${ }^{13} \mathrm{E}$. Landau, Differential and Integral Calculus (Chelsea, New York, 1951). 